\title{
IMPROVING THE PERFORMANCE OF 3-D SENSOR ARRAY BY ROTATION
}

\author{
Ayesha Naaz ${ }^{1}$ and Rameshwar Rao ${ }^{2}$ \\ ${ }^{1}$ Department of Electronics and Communication Engineering, \\ MuffakhamJah College of Engineering \& Technology,Hyderabad,India \\ ayeshanaaz10@gmail.com \\ ${ }^{2}$ Jawaharlal Nehru Technological University,Hyderabad,India \\ rameshwar_rao@hotmail.com
}

\begin{abstract}
In this paper, a method is proposed to improve the performance of 3-D array for DOA estimation. Cancellation of ground clutter received at the array (of sensors) is an important problem to be considered. The approach being addressed to reduced the clutter is based on rotating the 3-D array (a cubical array) by a fixed angle in all the three directions simultaneously. The computer simulations carried out showed that this method was capable of resolving closely spaced sources and also yielded optimum performance in achieving direction of arrivals. Compared to normal cubical array with the same number of elements, the rotated array provides a significant DOA accuracy. Further simulations were also carried out for various angles of rotation.
\end{abstract}

\section{KEYWORDS}

3-D array geometry, MUSIC, DOA, rotation

\section{INTRODUCTION}

Sensor array positioning has an important influence on the Direction Of Arrival (DOA) estimation performance of array processing. The desire for accurate DOA estimation can result in dealing with arrays with more number of elements/sensors. Though there exist high resolution algorithms to estimate the DOA ([3],[4],[5],[8]) it is inevitable to enlarge the array aperture or increase the number of elements to improve the performance of an array for a particular application.

Clutter returns pose problems in resolving targets/sources both in velocity and azimuth. Interference rejection capabilities of the array depend on its geometry. The antenna elements can be arranged in various geometries with linear, circular and planar arrays being very common ([2],[3],[4],[7]). Many of the higher-dimensional subspace based algorithms such as MUltiple SIgnal Classification (MUSIC) or Estimation of Signal parameters via Rotational Invariance Techniques (ESPRIT) can be used for estimation of DOA of which ESPRIT needs a special array geometry and cannot be applied to any arbitrary geometry [15]. Hence MUSIC algorithm has been applied to the geometry considered here.

The ability of achieving high performance is greatly influenced by the geometry of the array. There are basically three types of array geometries i.e., One Dimensional (1-D), Two Dimensional (2- D) and Three Dimensional (3- D). The $1 \mathrm{D}$ array geometry where usually a set of sensors are uniformly spaced in a line generally termed as a uniform linear array (ULA), have been dealt in [5]. In reference [9] a new algorithm was proposed for wide band sources impinging on ULA. In 2 D geometry, uniform rectangular arrays were investigated in [11], Uniform Circular arrays in [2] and [4] , L shape arrays in [3] and [8],triangular arrays 
were considered in [14] and [7]. For the 3 dimensional case sphere has been investigated using Matrix Pencil Method in [9] and [10].

In this paper, a cubical array (3-D geometry) with MUSIC algorithm is investigated for determining the direction of arrival (only azimuth angle determination taken up here) of signals incident on the sensor array. One dimensional array's suffers from 'East-West ambiguity' and 'north-south ambiguity'. In two Dimensional arrays the east west ambiguity is overcome but north south ambiguity still remains.

The limitations of the one dimensional and two dimensional arrays create problems for locating sources with much accuracy. A 3-D array in cubical configuration has been dealt with in this work to overcome the limitations of 1-D and 2-D arrays.

Assumption: In a typical DOA estimation problem it is often assumed that all the signals are arriving at the array at the same frequency which is assumed to be known. The array elements are then placed half the wavelength apart in the array.

The organization of the paper is as follows: Section 2 gives the evaluation algorithm used to find the direction of arrival. Section 3 depicts the signal model used and section 4 gives the simulation results obtained in MATLAB .Finally the conclusions are presented in section 5.

\section{THE MUSIC ALgORITHM}

Of the many high resolution algorithms MUSIC and ESPRIT are most commonly used. Here a cubical geometry is considered, the signals received at the sensors are the inputs to the MUSIC algorithm .The MUSIC algorithm is an implementation of the signal subspace approach to multiple source location and signal parameter estimation. To solve the multiple signal direction finding problem, the MUSIC technique makes use of the matrix of cross correlations between the individual sensor (antenna) signals. By means of an eigen structure analysis of the matrix, it is possible to isolate the complex vector subspace in which are single DOA vectors must lie. Then the correct DOA's can be found by determining which vectors in the array manifold lie (or intersect) in this subspace.

If there are $\mathrm{D}$ signals incident onto the array, the received input data vector at an $\mathrm{M}$ element array can be expressed as a linear combination of the $\mathrm{D}$ incident waveforms and noises.

If $r(t)$ is the array element received signal, $s(t)$ is the source signal, $n(t)$ is additive noise, the first array element is taken as reference array element, then receive signal at the kth array element is:

$$
r_{i}(\mathrm{t})=\sum_{k=1}^{D} a\left(\theta_{k}\right) s_{k}(t)+n_{i}(\mathrm{t})
$$

where, $\mathrm{i}=1,2, \ldots M$.The vector form of which can be written as

$$
r(t)=A s(t)+n(t)
$$

where: $\mathrm{r}(\mathrm{t})=\left[r_{1}(t), \quad \ldots \quad r_{M}(t)\right]_{M \times 1}^{T}$,

A the matrix of steering vectors is given as

$\mathrm{A}=\left[\begin{array}{lll}a\left(\theta_{1}\right) & \cdots & a\left(\theta_{D}\right)\end{array}\right]_{M \times D}$,

$\mathrm{s}(\mathrm{t})=\left[\begin{array}{lll}s_{1}(t) & \cdots & s_{D}(t)\end{array}\right]_{D \times 1}^{T}$ is the signal vector and

$\mathrm{n}(\mathrm{t})=\left[\begin{array}{lll}n_{1}(t) & \cdots & n_{M}(t)\end{array}\right]_{M \times 1}^{T}$ is a noise vector with components of variance $\sigma^{2}$.

The received vectors and the steering vectors can be visualized as vectors in an $M$ 
dimensional vector space.The covariance matrix of the array signal vector will be

$$
\mathrm{R}=\mathrm{AS} A^{H}+\sigma^{2} \mathrm{I}
$$

where $\mathrm{R}$ is the signal covariance matrix

$\mathrm{I}$ is an identity matrix,$\sigma^{2}$ is the variance of the additive noise and $\mathrm{S}$ denotes the Covariance matrix of the source signals.

MUSIC requires $S$ be non-singular, though even that assumption can be relaxed for certain geometries especially the linear uniform array.

Let $\quad \lambda_{1} \geq \lambda_{2} \geq \cdots \geq \lambda_{M}$ and $v_{1} \geq v_{2} \geq \cdots \geq v_{M}$ denote the eigen values of $\mathrm{R}$ and $\mathrm{A} \mathrm{S}$ $A^{H}$ respectively. From the structure of given by (4), these two sets of eigen values are related by

$$
\lambda_{i}=v_{i}+\sigma^{2}, i=1,2, \ldots M
$$

Assuming that the matrix A is of full column rank, which is generically the case, it follows that the rank of $\mathrm{A} \mathrm{S} A^{H}$ is $\mathrm{D}$, implying that the $\mathrm{M}-\mathrm{D}$ smallest eigenvalues of $\mathrm{A} \mathrm{S} A^{H}$ are equal to zero or equivalently, the smallest eigen value of $\mathrm{R}$ is equal to $\sigma^{2}$ with multiplicity $M-D$, i.e.,

$$
\lambda_{D+1}=\cdots=\lambda_{M}=\sigma^{2}
$$

The Eigen values $\lambda_{1}, \ldots, \lambda_{D}$ are known as the signal eigenvalues and $\lambda_{D+1}, \ldots, \lambda_{M}$ as the noise eigen values.

Let $e_{1}, e_{2}, \ldots, e_{M}$ denote the eigenvectors of R correspondingly to $\lambda_{1}, \ldots, \lambda_{M}$. Form matrices $E_{S}$ and $E_{N}$ as

$$
\begin{aligned}
E_{S} & =\left[\begin{array}{llll}
e_{1} & \ldots & e_{D}
\end{array}\right] \\
E_{N} & =\left[\begin{array}{llll}
e_{D+1} & \ldots . & e_{M}
\end{array}\right]
\end{aligned}
$$

$e_{1}, \ldots, e_{D}$ the signal eigenvectors, $e_{D+1}, \ldots, e_{M} \quad$ the noise eigenvectors. $E_{S}$ is the signal subspace and $E_{N}$ is the noise subspace.

By searching through all possible array steering vectors to find those which are orthogonal to the space spanned by the noise Eigen vectors $e_{D+1}, \ldots, e_{M}$ the DOAs $\theta_{1}, \theta_{2}, \ldots, \theta_{p}$ can be determined.

Then $a(\theta)^{*} E_{N} E_{N}{ }^{*} a(\theta)=0$ for $\theta$ corresponding to the DOA of incoming signal. In practice, $a(\theta)$ will not be precisely orthogonal to the noise subspace due to errors in estimating $E_{N}$. However the function

$$
P_{M U S I C}(\theta)=\frac{1}{a(\theta)^{H} E_{N} E_{N}{ }^{H} a(\theta)}
$$

known as the multiple signal classification (MUSIC) spectrum can be used to determine the DOA peaks.

This will assume a very large value when $\theta$ is equal to the DOA of one of the signals. The MUSIC algorithm, proposed by Schimdt[ ], first estimates a basis for the noise subspace $E_{N}$ and then determines peaks; the associated angles provide the DOA estimates. 


\section{ARRAY GEOMETRY}

The one dimensional and two dimensional arrays pose limitation for estimating the DOA's of impinging signals with much accuracy. The three Dimensional arrays overcome the east-west and north-south ambiguity of these array structures. Also a 3-D array provides better resolution as compared to one or two dimensional array even when they are added on with more elements (increased aperture area).

The geometry chosen in this paper is a cube. The array steering vector for the MUSIC algorithm is formed from the coordinates shown at the vertices of the cube where sensors are placed as shown in figure 1. The minimum number of elements employed to form a cubical array are eight.

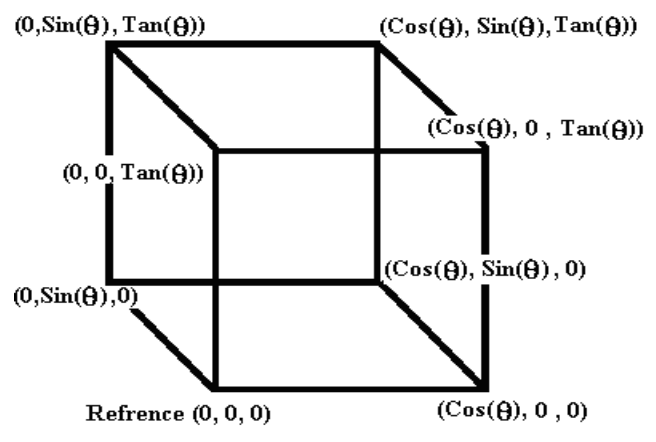

Figure 1. Cubical Array with sensors at its vertices

The cubical array configuration with eight sensors was used at the receiving terminal with spacing between each sensor as $\lambda / 2$, reference sensor at origin. The arriving signals were collected and MUSIC algorithm was applied to their co variances to obtain the DOA. The procedure was repeated by rotating the cubical array in all the three directions simultaneously by an angle $\theta$. The figure 2 shows the normal cubical array in red and rotated array in blue.

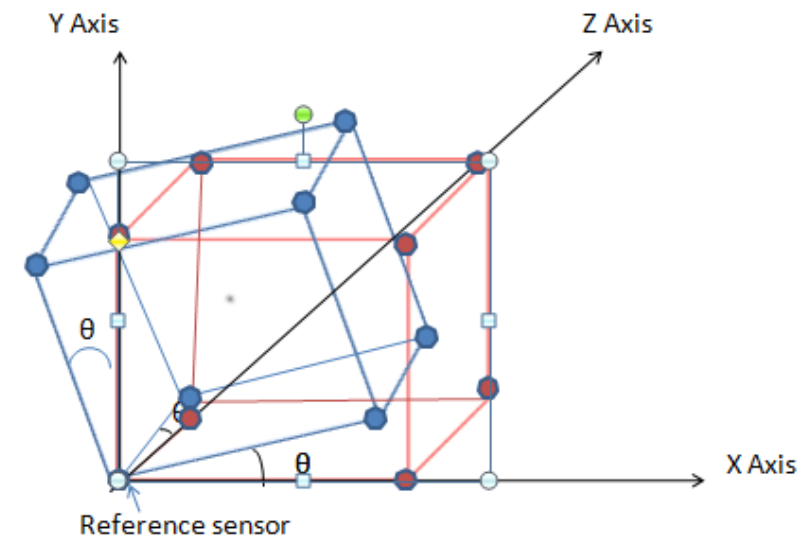

Figure 2: Cubical array normal (in red) and rotated (in blue)

\section{SimUlATION RESULTS}

In this section, the computer simulations are presented to verify the effectiveness of the rotation of the array. The simulations were performed in MATLAB and the results were obtained for three different signals at $30^{\circ}, 40^{\circ}$ and $46^{\circ}$ of same frequency. 
These simulations was conducted for

i) a cubical array with reference sensor at origin and no rotation,

ii) a cubical array rotated by $30^{\circ}$ angle in all the three directions and

iii) a cubical array rotated by $45^{\circ}$ in all the directions. It is observed that the $45^{0}$ rotated cubical array has better resolving capability than the other two.

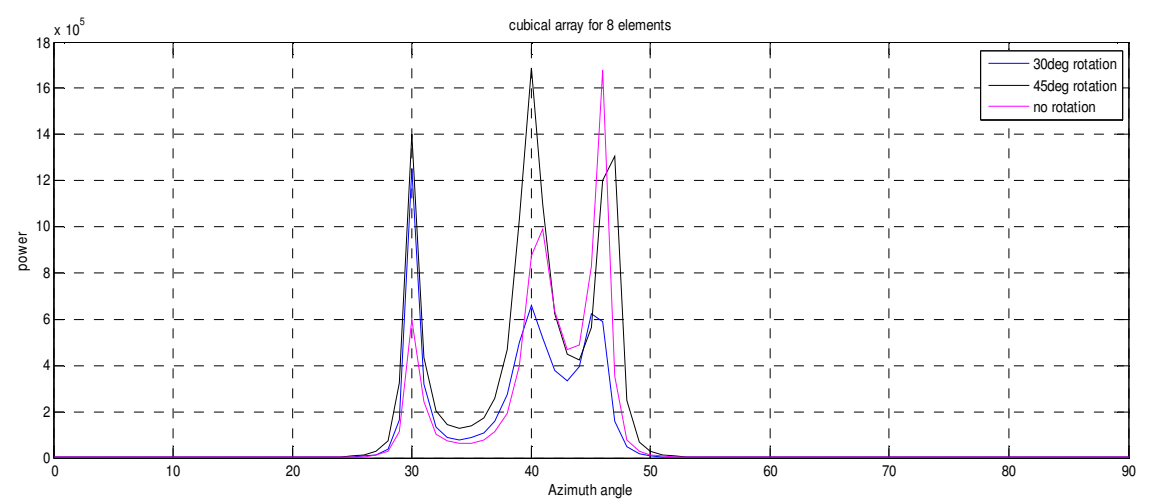

Figure 3: Results obtained for sources at angles $30^{\circ}, 40^{\circ}$ and $46^{\circ}$

The results show that the 3-D array with 45degree rotated geometry has better resolving capability as shown in figure 3 .

The simulations were also carried out for sources at angles $30^{\circ}, 40^{\circ}$ and $44^{\circ}$ and the simulations obtained as shown in figure 4 that the 45 degree rotated geometry is able to resolve the sources better than the other two geometries.

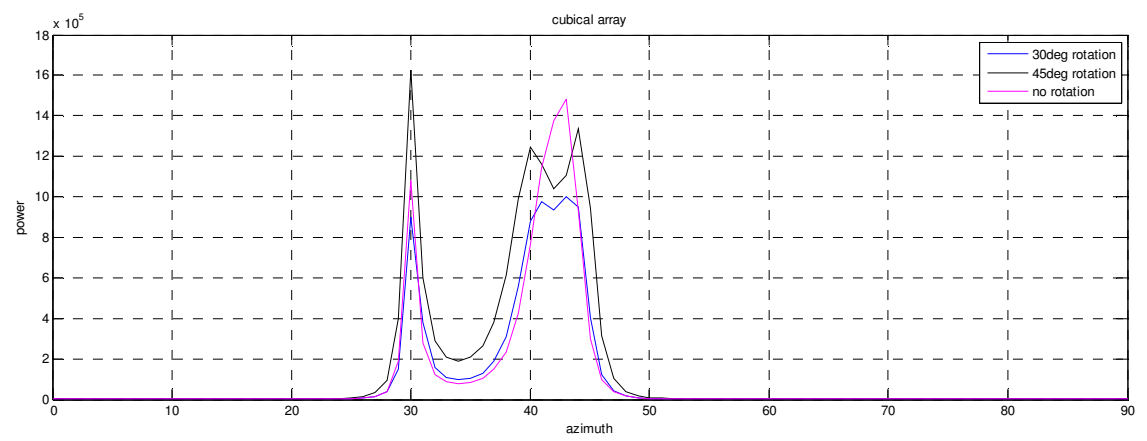

Figure 4: Results obtained for sources at angles $30^{\circ}, 40^{\circ}$ and $44^{\circ}$

\section{CONCLUSIONS}

A novel 3-D rotated geometry has been proposed and implemented on a cubical array for enhancing the resolution of the direction of arrival estimation. The paper presents a robust solution for accurate DOA estimation without increasing the number of array elements. The MUSIC algorithm has been chosen to present the performance of the geometry. Simulation results demonstrate that the proposed technique results in much better DOA estimation as compared to an array with same number of elements without rotation of the axes. Also the array presents better resolving capability when the rotated angle is $45^{\circ}$. The method also presents better resolving capability when the sources are closely placed. 
International Journal of Distributed and Parallel Systems (IJDPS) Vol.3, No.2, March 2012

\section{REFERENCES}

[1] R.O. Schmidt, "Multiple emitter location and signal parameter estimation", in: Proceedings of the RADC Spectral Estimation Workshop, Rome, NY, 1979, pp. 243-258.

[2] Naftali H ,Christos Christodolou "Uniform Circular Arrays for Smart Antennas",IEEE Antennas and propagation Magazine, Vol 47,No 4,August 2005.

[3] Chen Jain, Shuxun Wang,Lin Lin “2-D DOA Estimation by MEMP based on L Shape Array”, ICSP 2006 proceedings.

[4] M Zeytinoglu, J litva, J Qian "High resolution Direction Finding Using Circular Arrays",IEEE 1991.

[5] Xu-Dihua,Chen-Jianwen,Wu-You “ A Novel Subspace Coherent Signal Processing Algorithm for high resolution DOA Estimation”, ISPACS Proceedings 2006.

[6] H.L. Van Trees, Optimum Array Processing, Wiley, New York, 2002.

[7] Kainam T. Wong ,Michael D. Zoltowski "High Accuracy 2D Angle Estimation With Extended Aperture Vector Sensor Arrays" IEEE 1996.

[8] Nuri Yilmazer, Tapan K. Sarkar "2-D unitary matrix pencil method for efficient direction of arrival estimation”, Digital Signal Processing, Volume 16, Issue 6, November 2006, Pages 767 781.

[9] Monika Agrawal, S. Prasad "Estimation of directions of arrival of wideband and wideband spread sources”, Signal Processing, Volume 87, Issue 4, April 2007, Pages 614-622.

[10] Fulai Liua, Jinkuan Wanga, Ruiyan Duc, Ge Yua "Space-time matrix method for 2-D directionof-arrival estimation" Signal Processing 87 (2007) 101-106.

[11] Nizar Tayem, Hyuck M. Kwon "Azimuth and elevation angle estimation with no failure and no eigen decomposition” Signal Processing, Volume 86, Issue 1, January 2006, Pages 8-16.

[12] H. Krim, M. Viberg, Two decades of array signal processing research: the parametric approach, IEEE Signal Process. Mag. 13 (4) (July 1996) 67-94.

[13] R. Kumaresan, D.W. Tufts, "Estimating the Angles of Arrival of Multiple Plane Waves", IEEE Trans. AES, vol.1, p.134, 1983.

[14] Shi lei, m Sierra-Perez "Triangular Grid multi beam Array and beam Forming Network" UCAP 2007. The Second European Conference, 2007, Pages: 1 - 4.

[15] T B Lavate,V K Kokate, A M Sapkal ,'Performace analysisof MUSIC and ESPRIT DOA Estimation algorithms for adaptive array smart antenna in mobile communication”,IJCN, vol.2, issue 3, pages 152-158.

Authors

Prof Rameshwar Rao obtained his B.E Degree from Osmania University,M Tech and Ph D from IIT Bombay.

He is currently the Vice Chancellor of JNTUH and has held various positions of Dean Osmania University, Convener PGCET and professor Department of ECE OU. His experience spans across 34 years as R \& D engineer and as eminent teacher.His research interests include Detection and estimation theory, Information and coding theory,VLSI Design, VHDL modelling and synthesis.

Ayesha Naaz obtained the B.E degree from Osmania University, M Tech from JNTU Hyderabad and is currently pursuing $\mathrm{Ph} \mathrm{D}$ in the area of array signal processing. Currently Associate Professor in electronics she has a teaching experience of 13 years. Her research interests include VHDL modelling and synthesis, Signal processing. 